\title{
HIGH-SPEED WAVELENGTH ENCODED MULTIPLE-CHANNEL OPTICAL BUSES FOR LAN
}

\author{
Chin-Chou Chen**, Lon A. Wang* and Sy-Yen Kuo \\ Department of Electrical Engineering, National Taiwan University \\ Taipei, Taiwan, R. O. C.
}

\begin{abstract}
Scalable, high-speed, wavelength encoded multiple-channel optical buses (WEMCOB) employing wavelength division multiplexing (WDM) technologies are proposed to alleviate link speed constraints, wiring complexity and to increase power budget economically for a local area network (LAN). A hierarchical topology is adopted in the network where a dual uni-directional WEMCOB with separate control and data channels composes the backbone network, and unidirectional tree-based WEMCOB's with centralized arbiters constitute the sub-networks. We show that by utilizing today's opto-electronic technology a total transmission capacity of several tens of gigabits per second $(\mathrm{Gb} / \mathrm{s})$ can be obtained for a WEMCOB-based LAN.
\end{abstract}

\section{INTRODUCTION}

With the advent of more complex and advanced broadband services, the need for a high-speed LAN has become quite urgent. A network delivering such broadband services generally requires high throughput and tight delay constraints particularly for real time traffic [1]. An appropriate choice should therefore be made for each transmission technique, network topology, and access protocol.

Among various transmission media electrical cables are not capable of $\mathrm{Gb} / \mathrm{s}$ data transport because of high transmission loss and crosstalk limitations. In contrast, at such rates data can be transmitted by optical fibers over longer distance with less power consumption than by electrical cables. However, most of today's digital transmission systems of a network utilize a bit-serial transmission with multiplexing/demultiplexing technique for data transport. As data rates go beyond than a few $\mathrm{Gb} / \mathrm{s}$, electronic control circuitry becomes quite involved and is yet mature. Even worse, extra framing and encoding required in a bit-serial link usually constitute a large portion of overhead. The bit-serial trans-mission scheme is therefore not expected practical in such high-speed data links. In contrast, a word-in-parallel transmission scheme requiring no extra framing may appear as an attractive alternative. Two approaches namely multi-copper and multifiber transmissions have been taken to implement a word-in-parallel scheme[2-4, 16]. Recently, however, other alternatives employ WDM have been proposed to transport data in a fully parallel form through $a$ single fiber $[5,6]$. In this paper, we propose a novel Wavelength Encoded Multiple-Channel Optical Bus (WEMCOB) for a high-speed LAN. The wavelength-per-bit encoding is based on WDM technologies, and all bits in separate low-speed control and high-speed data channels are transmitted in parallel.

Acknowledgment: This research was supported by the National Science Council, Taiwan,

R. O. C., under the Grants NSC 82-0408-E002-021 and NSC 83-0417-E002-005.

* The author is also with the Institute of Electro-Optical Engineering, National Taiwan University. ** The anthor is also with the Telecommunication Laboratories, Ministry of Transportation and Communications, $R$. O. C.
Many traditional network topologies such as ring, star, bus, or their combinations can be configured to comprise a fiber LAN. Among them a dual bus offers three appealing features. First, the sharing bandwidth doubles and the network reliability increases due to the duplex nature of two opposite directional buses. Secondly, all nodes can be easily added to or dropped from the network. Thirdly, a number of standardized high-performance distributed protocols, e.g. IEEE 802.6 DQDB (Distributed Queue Dual Bus) protocol [8], can be implemented to deal with network issues such as fairness, band-width balancing, congestion control and so on. There are two major issues, however, regarding a dual bus topology. First, the total band-width increases proportionally to the demand of broadband service, resulting in a bottleneck in a bit-serial link. Secondly, due to the distributive nature of a dual bus, the network size is limited by the power budget available from the existing opto-electronic devices. We propose a hierarchical architecture to alleviate these issues. The architecture consists of a main dual bus as the back-bone network and many scalable tree-based buses as the sub-networks.

The rest of this paper is arranged as follows. In Section II we will first review several high-speed multiple-channel optical buses reported so far. The encoding technique used in our WEMCOB for separate control and data channels is described in Section III. Then, in Section IV, based on the WEMCOB's a hierarchical topology for LAN is proposed. The consideration of power budget and system complexity for building the WEMCOB-based LAN is detailed in Section V. Finally, in Section VI we conclude our study.

\section{HIGH-SPEED MULTIPLE-CHANNEL OPTICAL BUSES}

We categorize the reported high-speed multiple-channel optical buses into two classes: one is of multiple-fiber optical bus (MFOB), and the other is of wavelength encoded multiple-channel optical bus (WEMCOB).

\section{A. Multiple-Fiber Optical Bus (MFOB)}

Two kinds of configuration have been studied in this class. The first kind is to transmit a number of independent bit-serial channels simultaneously by multiple fibers but asynchronously [3]. Each channel has its own transmitter, receiver and clock retiming devices. Major efforts and results of the MFOB research so far are technological developments for integrating multiple fibers with optoelectronic arrays and large volume reduction in interconnects, respectively. The second kind is to transmit data bits in a fully parallel form. The MODLINK II developed by AT\&T serves as an example[4]. It consisted of eight synchronized data channels each operating at speeds up to $500 \mathrm{Mb} / \mathrm{s}$ either in a continuous or in a bursty mode. The implementation by multiple fibers was expected to be cheaper than by multi-copper. 


\section{B. Wavelength Encoded Multiple-Channel Optical Bus (WEMCOB)}

In contrast to the MFOB's, a WEMCOB can have a big saving on fiber utilization since data bits are transmitted in parallel through $a$ single fiber. Each bit of the parallel data (word or byte) is encoded with a separate wavelength. These wavelengths are combined by a WDM multiplexer (mux), and then transmitted through a single-mode fiber. At the receiver side, a WDM de-multiplexer (demux) separates the received optical signals by wavelength. The signals are then converted into electrical signals of their original formats.

Several applications utilizing WEMCOB's have been demonstrated. For example, a tightly coupled architecture for computing was proposed in which each bit of routing and data information within a packet was encoded with a separate wavelength. However, the number of wavelengths available from the current WDM technology is yet large enough to support a big data packet containing, for example, a few hundred bits. Another example is a scalable interconnection architecture called Virtual Bus which exploited the feasibility of using WDM technology to alleviate interconnection constraints [6]. In the Virtual Bus, each data packet was partitioned and simultaneously transmitted by wavelength in sequential. The Virtual Bus approach may solve the wavelength availability problem encountered by the system described in Ref. 5 particular when a large packet is used.

\section{Comparisons of $M F O B$ and $W E M C O B$}

A common advantage for both MFOB and WEMCOB is the information transfer rate can be increased without varying the terminal circuits' speed due to the fact that, as opposed to a bit-serial transmission system, extra encoding and framing are not required. Since a WEMCOB encodes all bits in wavelengths rather than in fibers, it offers the following unique advantages: (i) reduced wiring complexity, (ii) reduced optical splitter complexity, (iii) fewer dispersion compensators, and (iv) fewer optical amplifiers for loss compensation. The first three advantages are quite obvious because only a single fiber is used in a WEMCOB, while the last one will be discussed in Section V-B. However, two issues regarding a WEMCOB shall be considered: an increased complexity of multi-wavelength transceivers and an increase of insertion loss due to WDM mux/demux. The former issue is expected to be relieved by the continued breakthrough of transceiver array technologies while the latter one resulting in a power budget concern will be discussed in Section V.

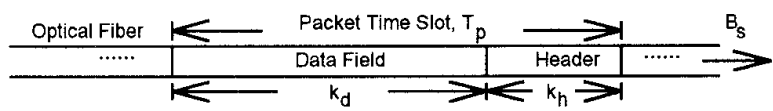

(a)
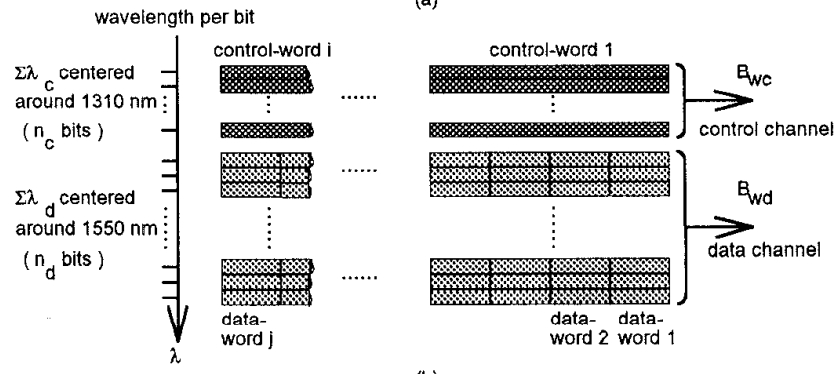

(b)

Fig. 1 Encoding techniques in a paacket being transmitted (a) for a serial optical link at a bit rate Bs; (b) for a WEMCOB where control channel are at a low-speed rate BwC and data channel at a high-speed rate Bwd.

\section{A NOVEL ENCODING TECHNIQUE FOR WEMCOB}

For most of today's packet switching technologies, a packet format contains two fields: a header field and a data field. The header field carries the control information while the data field contains the data to be transported. In general, the bit length of a header field is much shorter than that of a data field, and only the contents in the header field are to be processed in a switching node. The switching node of a bit-serial link must then be operated at the peak rate of a transmission line because the content of each packet is transmitted bit-by-bit sequentially. As the operation speed increases beyond several $\mathrm{Gb} / \mathrm{s}$, electronics becomes more and more involved, expensive and immature. Therefore, it becomes attractive and operationally efficient to divide the switching rate into two components: a high-speed data rate and a low-speed header rate. A similar concept called field-coding was proposed in a serial optical distribution channel [9]. In our WEMCOB, however, this field-encoding is employed in a fully parallel form.

Each packet being transmitted in our WEMCOB is divided into a control and a data channels as shown in Fig. 1. The pipelined control stream of the control channel is encoded with $n_{c}$ wavelengths equally spaced and centered around $1310 \mathrm{~nm}$. The control channel is operating at a low-speed rate $B_{w c}$. Similarly, the pipelined data stream of the data channel is encoded with $\mathbf{n}_{d}$ wavelengths but centered around $1550 \mathrm{~nm}$ and is operating at a high-speed rate $B_{w d}$. To show the WEMCOB employing a field-encoding technique can alleviate link speed constraints, we assume that a packet operating at a bit rate of $B_{S}$ contains a $k_{h}$-bit header field and a $k_{d}$-bit data field as shown in Fig. 1(a). After field-encoding all bits in both control and data channels are transmitted in parallel but at different speeds as shown schematically in Fig. 1(b). The control rate $B_{w c}$ and the data rate $B_{w d}$ can be expressed as

$$
B_{w c}=\left(\frac{k_{h} \cdot B_{s}}{k_{h}+k_{d}}\right) \cdot \frac{1}{n_{c}} \text { and } B_{w d}=\left(\frac{k_{d} \cdot B_{s}}{k_{h}+k_{d}}\right) \cdot \frac{1}{n_{d}},
$$

respectively.

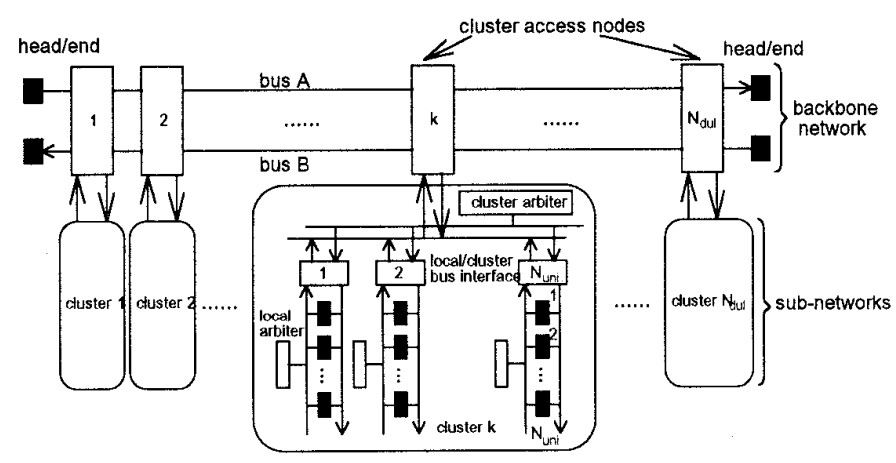

Fig. 2 A hierarchical WEMCOB architecture for local area network.

\section{BUILDING A LAN BASED ON THE WEMCOB'S}

In this section, we propose a hierarchical WEMCOB-based LAN to lift the network size limitation of a single-level bus network. Shown in Fig. 2 are a dual uni-directional WEMCOB and tree-based WDM clusters. The wavelength encoding technique as well as a distributed 
control scheme are employed to construct a dual uni-directional WEMCOB and tree-based WDM clusters. The dual uni-directional WEMCOB performs backbone switching, while the clusters identical to those proposed in the Virtual Bus function as in-campus or inbuilding sub-networks [6]. The two networks is interconnected by a cluster access node which performs media access control (MAC) protocols, interworking interface and data switching functions. Details are described as follows.

\section{A. Tree-Based WEMCOB Cluster}

The functional block of the WEMCOB local/cluster bus networking is shown in Fig. 3. Each node consists of a transmitter module, a receiver module, optical multiplexers, and an electrical processing unit. Parallel data and control signals from the electronic processing unit are electrically multiplexed and fed into a transmitter module. The transmitter module consists of an integrated multiplewavelength laser array with electrical drivers. Control and data channels are transmitted in different wavelengths as described in Section III. These signals are optically multiplexed by a WDM component, and then coupled into an optical fiber by an optical splitter. The protocol and the routing control are completed by a fast tree VLSI arbiter [7]. Details of the performance analysis and the technical feasibility of building such a cluster are described elsewhere $[6,7]$.

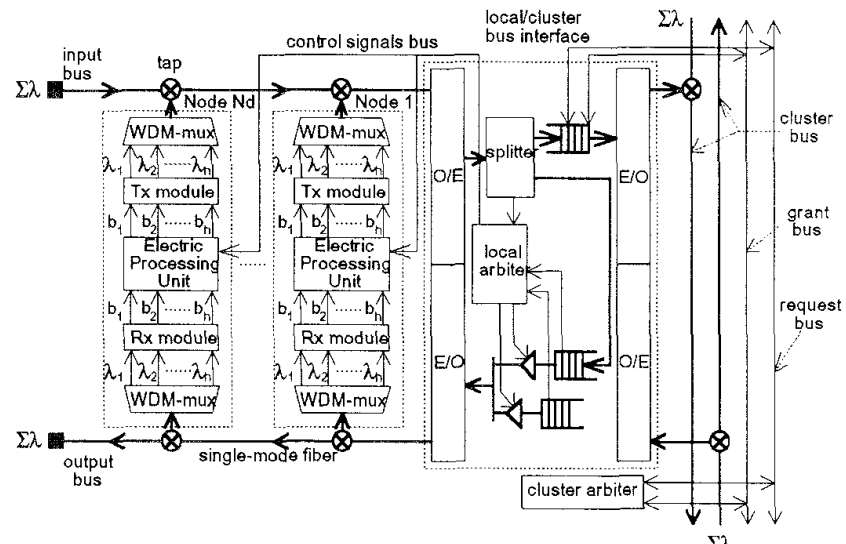

Fig. 3 One of the tree-based configration of a WEMCOB cluster in the sub-network.

\section{B. Dual Uni-Directional WEMCOB}

The encoding technique mentioned previously is applied to a DQDB packet of the proposed WEMCOB. The packet typically contains a 5-octet header and a 48-octet data fields, and each content of the fields is encoded with 8 and 16 wavelengths, respectively. The networking protocol of the WEMCOB is similar to a DQDB which is one of the international standards of MAN [8]. Faimess and bandwidth balancing are two important issues when using DQDB, and many methods have been proposed to make a more efficient and fair networking such as the use of erasure nodes [10] or the bandwidth balancing scheme [11], etc. It is therefore expected that with some modifications the existing DQDB protocols may as well be implemented in the dual uni-directional WEMCOB. The DQDB protocol and its control are carried out by the MAC in a cluster access node Fig. 4 depicts only a half portion of such a cluster access node while the other half is identical but in the opposite direction. Empty slots generated sequentially by the head/end nodes are detected and used by the successive nodes. The input packet is tapped out from a fiber to a cluster access node, and then divided into control and data channels by a $1.3 \mathrm{~V} / 1.55 \mu \mathrm{m}$ WDM-demux. These two channels, one at $1.31 \mu \mathrm{m}$ and the other at $1.55 \mu \mathrm{m}$, are further divided into finer channels, i.e. with a narrower channel spacing, before being processed by the MAC processor and the data queues unit. The slot information in the MAC processor is used to decide whether to receive or to send a packet. The data queue and interface module is used (i) to receive and to buffer an incoming packet; (ii) to perform interworking function between the dual WEMCOB and the clusters. Since $1.31 \mu \mathrm{m}$ and $1.55 \mu \mathrm{m}$ signals are multiplexed in a single fiber, depending on node-to-node distance and transmission speed some dispersion compensators may be required to maintain an acceptable bit error rate for both channels.

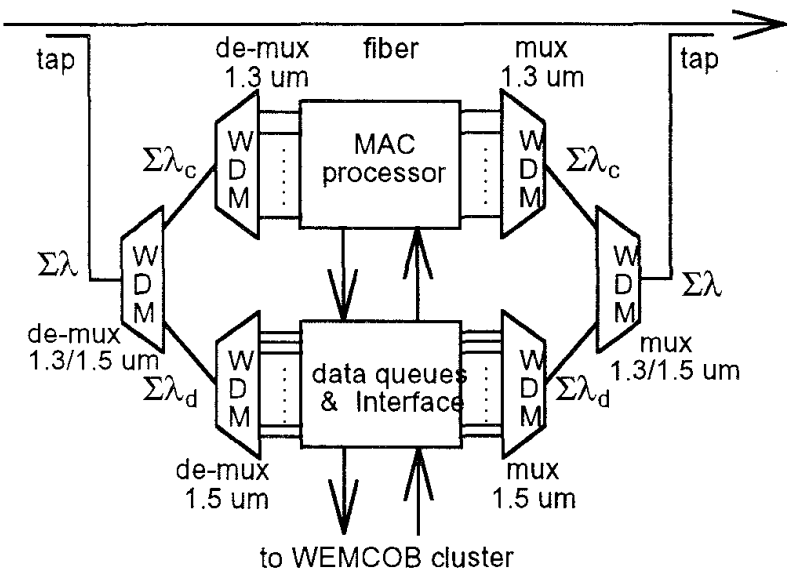

Fig. 4 Cluster access node structure of a dual uni-directional WEMCOB.

\section{POWER BUDGET AND SYSTEM COMPLEXITY OF THE WEMCOB-BASED LAN}

\section{A. Power Budget Analysis}

In our dual bus network, the worst power budget occurs in the last node after the signal power has been successively tapped out in the previous nodes. The optical power budget thus sets a limit on the maximum number of nodes that the network can have. For a unidirectional WEMCOB, the overall loss mechanisms of each node can be modeled as shown in Fig. 5. The insertion loss of a splitter in the "bar" and in the "cross" conditions are denoted as $L_{b a r}$ and $L_{\text {cross' }}$ respectively. $L_{c}$ and $L_{W D M}$ are the insertion loss of a directional coupler and of a WDM mux/demux. All the losses are expressed in $\mathrm{dB}$. The total loss of an n-node WEMCOB, denoted as $\mathrm{L}_{\text {total, }}$, can be therefore expressed as

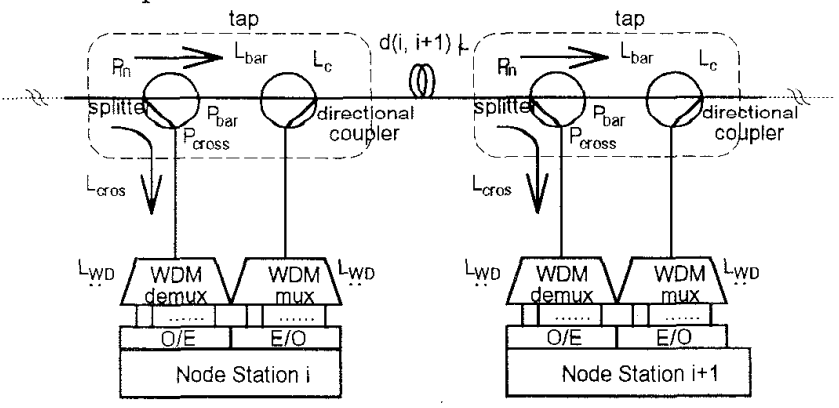

Fig. 5 Transmission loss model of a linear WEMCOB. 


$$
\begin{aligned}
\mathrm{L}_{\text {total, } W}=(\mathbf{n}-1) \cdot \mathrm{L}_{\mathrm{c}} & +\mathrm{L}_{\text {cross }}+(\mathbf{n}-\mathbf{2}) \cdot \mathrm{L}_{\text {bar }} \\
& +\sum_{\mathrm{i}=1}^{\mathrm{n}-1} \mathrm{~d}(\mathrm{i}, \mathrm{i}+1) \cdot \mathrm{L}_{\mathrm{f}}+2 \cdot \mathrm{L}_{\mathrm{WDM}}
\end{aligned}
$$

where $L_{f}$ is the fiber loss in $d B / k m$ and $d(i, i+1)$ is the fiber distance from node $i$ to node $i+1$. In addition, the insertion loss of a splitter defined as the sum of the excess loss and the coupling loss in each "bar" and "cross" condition can be expressed as

$$
\mathrm{L}_{\text {bax }}=\mathrm{L}_{\mathrm{ex}}+\left(-10 \log \frac{\alpha}{1+\alpha}\right) \text { and } \mathrm{L}_{\text {eross }}=\mathrm{L}_{\mathrm{ex}}+\left(-10 \log \frac{1}{1+\alpha}\right) \text {, }
$$

respectively. $L_{e x}$ is the excess loss of a splitter, and $\alpha$ is the splitting ratio defined as the output power in the "bar" condition divided by that in the "cross" condition. $\mathrm{L}_{\text {total, }}$ can thus be minimized by optimizing the splitting ratio for a total number of stations $n$ by substituting Eq. (3) into Eq. (2), and by differentiating Eq. (2) with respect to $\alpha$. The optimum splitting ratio, $\alpha_{\text {opt }}$, is found to be $\alpha_{\text {opt }}=n$ 2. If each splitter has an optimum splitting ratio $\alpha_{\text {opt }}$, and two adjacent nodes have the same distance $d$, the minimum total loss can therefore be found as

$$
\begin{array}{r}
L_{\text {total, } W}=(n-1) \cdot\left(L_{\text {ex }}+L_{c}\right)+\left[-10 \log \frac{(n-2)^{n-2}}{(n-1)^{n-1}}\right] \\
+(n-1) \cdot d \cdot L_{f}+2 \cdot L_{W D M} .
\end{array}
$$

It is noted that Eq. (4) is the summation of the splitter insertion loss, the splitter splitting loss, fiber attenuation loss and the WDM mux/demux insertion loss. Note that only the WDM insertion loss is independent of the number of nodes. It is apparent that the insertion loss of a splitter and the fiber loss are two deciding factors for a minimum total loss when the number of nodes becomes large. Fig. 6 shows the relationship between the power budget and the number of nodes that can be implemented in a WEMCOB for various insertion losses of splitters and fiber losses. The insertion loss of $-7 \mathrm{~dB}$ for a WDM mux/demux [12], and a node-to-node distance of $100 \mathrm{~m}$ are assumed. We find from Fig. 6 that as the splitter insertion loss or fiber loss increases, the nodes that a WEMCOB can accommodate decrease dramatically. Also noted is that although the total WDM mux/demux insertion loss is not node number dependent, the loss incurs a large decrease in power budget, $14 \mathrm{~dB}$ in Fig. 6 . This suggests the significance of realizing a WDM mux/demux with low insertion loss, which may be feasible by employing an integrated grating [13]. Otherwise, optical amplifiers need to be installed for loss compensation. Depending on the working wavelength regions, a possible candidate for optical amplifier may be an erbium-doped fiber amplifier (EDFA, $\sim 1.55 \mu \mathrm{m}$ ), a praseodymium-doped fluoride fiber amplifier $(\sim 1.31 \mu \mathrm{m})$, or a semiconductor amplifier (both $1.31 \mu \mathrm{m}$ and $1.55 \mu \mathrm{m})$.

\section{B. Consideration of Using Optical Amplifiers}

As analyzed previously, the number of nodes in a WEMCOB is limited by the transmission and the insertion losses, the use of optical amplifiers shall be considered to boost up power budget. Since an optical amplifier is still quite costly, it is important to estimate how many amplifiers are required in a WEMCOB, and then compare with an MFOB. For simplicity and without the loss of generality, we take the data channel of a WEMCOB as an example for amplification. The EDFA's used in both MFOB and WEMCOB are assumed to have the same unsaturated and equalized gain over the entire wavelengths of interest. We further assume that a WEMCOB has all the same losses as an MFOB expect that an additional WDM mux/demux insertion loss needs to be considered in a WEMCOB. The minimum total loss of an MFOB, $\mathrm{L}_{\text {total, } \mathrm{F}}$, is therefore related to that of a WEMCOB, $\mathrm{L}_{\text {total,w }}$ (Eq. (4)), by

$$
\mathrm{L}_{\text {total }, \mathrm{F}}=\mathrm{L}_{\text {total }, \mathrm{W}}-2 \cdot \mathrm{L}_{\mathrm{WDM}} \text {. }
$$

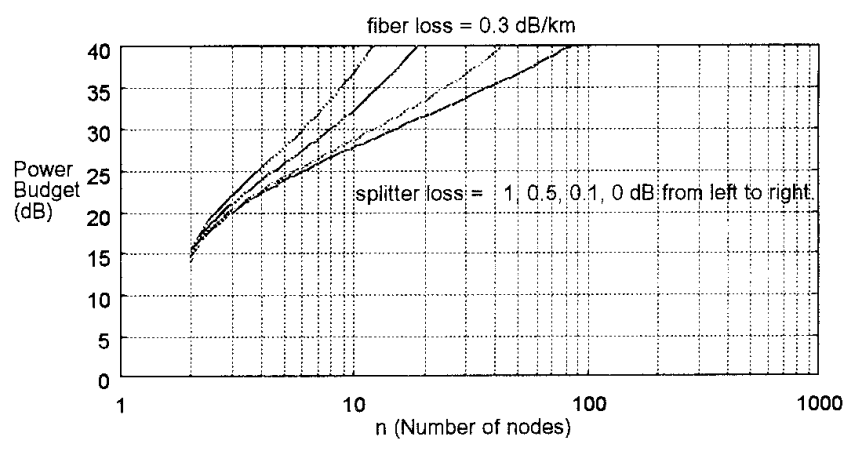

(a)

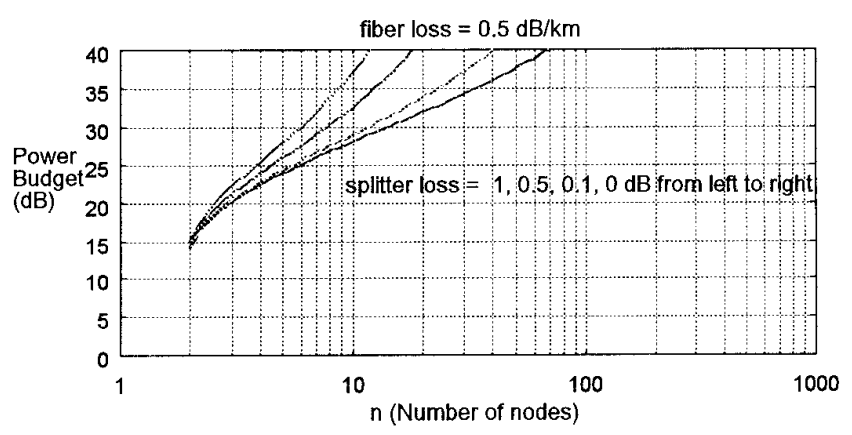

(b)

Fig. 6 The relationship between the power budget and the number of nodes as a function of splitter loss, where $\mathrm{L}_{\text {WDM }}=7 \mathrm{~dB}$ and $\mathrm{d}=100 \mathrm{~m}$ for $\mathrm{L}_{\mathrm{f}}=$

(a) 0.3 and (b) $0.5 \mathrm{~dB} / \mathrm{km}$, respectively.

To estimate the number of optical amplifiers used for loss compensation in both MFOB- and WEMCOB-based LAN's, we consider a case that a packet of $\mathbf{k}$ bits is to be transmitted. It is apparent that in an MFOB LAN, a total of $k$ optical amplifiers are required since $\mathrm{k}$ fibers are physically utilized for transmission. In contrast, a k-wavelength WEMCOB LAN only requires a single optical amplifier since $\mathrm{k}$ signal-encoded wavelengths are simultaneously amplified. In reality, although each wavelength may have different gain due to the non-uniform gain profile of an EDFA, significant achievements have been made by utilizing various techniques or algorithms for gain equaliz-ation $[14,15]$. In addition, unlike long-haul transmission the spanned area of a LAN is typically within several kilometers, cascading of optical amplifiers for in-line 
signal amplification may not be necessary, which further simplifies gain equalization. As described previously, we can therefore assume that each signal (or wavelength) in a WEMCOB will experience the same gain as that in an MFOB. The number of nodes supported by a WEMCOB and an MFOB with optical amplifiers can then be calculated from both Eq. (4) and Eq. (5) by replacing the total loss with the gain value of an optical amplifier. For illustration, we now consider an example in which a 1000-node LAN to be built in a 16parallel-channel form. Each channel is operated at $622 \mathrm{Mb} / \mathrm{s}$, and a power budget of $30 \mathrm{~dB}$ is assumed for each transceiver at this bit rate [17]. We further assume that the distance between two adjacent nodes is $100 \mathrm{~m}$ and each optical amplifier has $40 \mathrm{~dB}$ gain. Calculations show that the number of amplifiers required for a WEMCOB and for an MFOB are 23 and 144 , respectively, assuming that the splitter loss, fiber loss and WDM loss are $0.1 \mathrm{~dB}, 0.3 \mathrm{~dB} / \mathrm{km}$ and $7 \mathrm{~dB}$. It shows clearly an MFOB requires more optical amplifiers than a WEMCOB does.

\section{System Complexity}

Let the number of nodes connected to the dual uni-directional WEMCOB and to the WEMCOB cluster be $\mathrm{N}_{\text {dul }}$ and $\mathrm{N}_{\text {uni' }}$, respectively, as depicted in Fig. 2. Depending on bit rates and total losses, $\mathrm{N}_{\text {dul }}$ and $\mathrm{N}_{\text {uni }}$ can be determined from each individual power budget. The total number of nodes supported by the WEMCOB-based LAN can then be expressed as

$$
\mathrm{N}_{\text {total }}=\mathrm{N}_{\mathrm{dul}} *\left(\mathrm{~N}_{\text {uni }}\right)^{\mathrm{L}}
$$

where $\mathrm{L}$ is the level of the tree in a WEMCOB cluster. It is by the tree level expanding the scalability of our WEMCOB-based LAN can be achieved. For illustration, we assume that a WEMCOB can support $\mathrm{N}$ nodes and all WEMCOB's in the LAN are identical, then for a dual uni-directional WEMCOB with $\mathrm{N}$ two-level tree-based WEMCOB clusters the total number of nodes can reach $\mathrm{N}^{3}$ (i.e. $\mathrm{N}_{\mathrm{dul}}=\mathrm{N}_{\mathrm{uni}}=\mathrm{N}$, $\mathrm{L}=2$ ). If the WEMCOB-based LAN is operated at $622 \mathrm{Mb} / \mathrm{s}$ datachannel rate and at $129 \mathrm{Mb} / \mathrm{s}$ control-channel rate, the total bandwidth is equivalent to a serial dual bus of $\sim 22 \mathrm{~Gb} / \mathrm{s}(622 \mathrm{Mb} / \mathrm{s} * 16 * 53 / 48$ * $2=21.98 \mathrm{~Gb} / \mathrm{s})$. For such capacity, a total of 1000 nodes can be built in the WEMCOB-based LAN without installing optical amplifiers by assuming that a transceiver-array has a power margin of $29 \mathrm{~dB}$ and a splitter loss is $0.1 \mathrm{~dB}$ as shown in Fig. 6(a).

\section{CONCLUSIONS}

We have proposed a scalable, high-speed, fully parallel WEMCOB-based LAN which can alleviate link speed constraints, wiring complexity and can economically increase power budget when compared to an MFOB-based LAN. The LAN is composed of a dual WEMCOB as the backbone network, and uni-djrectional tree-based WEMCOB clusters as the sub-networks. In the WEMCOB's, a novel encoding technique which combines WDM and field-coding has been proposed for packet switching. We have shown that such a technique can effectively reduce the speeds of both the control and the data channels so that a practical implementation for a high-speed LAN is feasible using current opto-electronic technologies. The power budget analysis identifies that splitter insertion loss and fiber loss are two dominant factors in determining the number of nodes that a WEMCOB can accommodate. When optical amplifiers are employed for loss compensation, calculations indicate that a WEMCOB-based
LAN may have a big saving for the optical amplifiers over an MFOBbased LAN. It is estimated that the WEMCOB-based LAN can be practically implemented to serve a thousand nodes with a total capacity of several tens of $\mathrm{Gb} / \mathrm{s}$.

\section{REFERENCE}

[1] CCITT Draft Recommendations for B-ISDN, 1990.

[2] M. L. Loeb and G. R. Stilwell," High-Speed Data Transmission on an Optical Fiber Using Byte-Wide WDM System," J. Lightwave Technol, vol. 6, pp. 1306-1311, 1988.

[3] N. Fujimoto et al," Gigabit-per-second optical parallel transmitter/receiver module with fully integrated GaAs MESFET LSI chips," in $O F C^{\prime} 93$, paper ThC1, 1993.

[4] Y. Ota and R. G. Swartz," Multichannel 4-Gbit/s (500-Mbit/s-perchannel) parallel optical data link," in $O F C^{\prime} 93$, paper ThC2.

[5] D. J. Blumenthal et al," Demonstration of a Deflection Routing $2 \mathrm{x}$ 2 Photonic Switch for Computer Interconnections," IEEE Photon. Technol. Lett., vol. 4, pp. 169-173, 1992.

[6] L. A. Wang and K. C. Lee," An Opto-Electronic Virtual Bus Using WDM and VLSI technologies for Interconnection Networks," in Proc. Conf. ICC'92, Chicago, LA, pp. 888-894, 1992.

[7] K. C. Lee," A Virtual Bus Architecture for Dynamic Parallel Processing," IEEE Trans. Paral. and Distribut. System, vol. 4, pp. 121-130, 1993.

[8] IEEE 802.6 Working Group, " IEEE standard 802.6: Distributed Queue Dual Bus (DQDB) subnetwork of a metropolitan area network (MAN)," Final Draft D15, approve by IEEE Standards Board on Dec. 6, 1990.

[9] Z. Haas and R. D. Gitlin," Optical Distribution Channel: An "Almost-all" Optical LAN based on the Field-coding Technique", in Proc. Conf. INFOCOM'92, pp. 57-62, 1992.

[10] P. Breault and V. Phung, " DQDB performance improvement with erasure nodes," Contribution 802.6-90/21 to the IEEE 802.6 Working Group, Mar. 1990.

[11] E. L. Hahne et al," DQDB Networks with and without Bandwidth Balancing," IEEE Trans. on Commun., vol. 40, no. 7 July, 1992.

[12] Data quoted from a commercially available grating-based WDM component, e.g. from Instruments SA products.

[13] J. B. D. Soole et al," Monolithic InP/InGaAsP/InP grating spectrometer for the $1.48-1.56 \mu \mathrm{m}$ wavwlength range," Appl. Phys. Lett., vol. 58, pp. 1949-1951, 1991.

[14] A. R. Chraplyvy et $a l, "$ Equalization in amplified WDM Lightwave Transmission Systems," IEEE Photon. Technol. Lett., vol. 4, pp. 920-922, 1992.

[15] E. L. Goldstein et al," Inhomogeneously Broadened FiberAmplifier Cascade for Wavelength-Multiplexed Systems," IEEE Photon. Technol. Lett., vol. 5, pp. 543-545, 1993.

[16] M. L. Loeb and G. R. Stilwell," An Algorithm for Bit-Skew Correction in Byte-Wide WDM Optical Fiber Systems," IEEE J. Lightwave Technol., vol. 8, pp. 239-242, 1990.

[17] Y. Akahori et al, "A $622 \mathrm{Mb} / \mathrm{s}$ Monolithically Integrated InGaAs/InP Four-Channel p-i-n FET Receiver Array," IEEE Photon. Technol. Lett., vol. 4, pp. 470-472, 1992. 